Louisiana State University

LSU Digital Commons

Faculty Publications

Department of Physics \& Astronomy

$1-1-2005$

\title{
Calculation and manipulation of the chirp rates of high-order harmonics
}

\author{
M. Murakami \\ Louisiana State University \\ J. Mauritsson \\ Louisiana State University
}

A. L'Huillier

Lunds Tekniska Högskola

K. J. Schafer

Louisiana State University

M. B. Gaarde

Louisiana State University

Follow this and additional works at: https://digitalcommons.Isu.edu/physics_astronomy_pubs

\section{Recommended Citation}

Murakami, M., Mauritsson, J., L'Huillier, A., Schafer, K., \& Gaarde, M. (2005). Calculation and manipulation of the chirp rates of high-order harmonics. Physical Review A - Atomic, Molecular, and Optical Physics, 71 (1) https://doi.org/10.1103/PhysRevA.71.013410

This Article is brought to you for free and open access by the Department of Physics \& Astronomy at LSU Digital Commons. It has been accepted for inclusion in Faculty Publications by an authorized administrator of LSU Digital Commons. For more information, please contact ir@lsu.edu. 


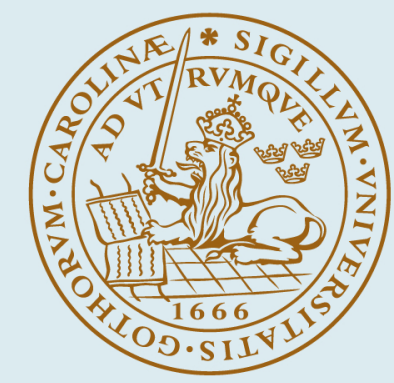

\title{
LUND UNIVERSITY
}

\section{Calculation and manipulation of the chirp rates of high-order harmonics}

\author{
Murakami, M; Mauritsson, Johan; L'Huillier, Anne; Schafer, KJ; Gaarde, Mette
}

Published in:

Physical Review A (Atomic, Molecular and Optical Physics)

DOI:

10.1103/PhysRevA.71.013410

2005

Link to publication

Citation for published version (APA):

Murakami, M., Mauritsson, J., L'Huillier, A., Schafer, KJ., \& Gaarde, M. (2005). Calculation and manipulation of the chirp rates of high-order harmonics. Physical Review A (Atomic, Molecular and Optical Physics), 71(1).

https://doi.org/10.1103/PhysRevA.71.013410

\section{Total number of authors:}

5

\footnotetext{
General rights

Unless other specific re-use rights are stated the following general rights apply:

Copyright and moral rights for the publications made accessible in the public portal are retained by the authors and/or other copyright owners and it is a condition of accessing publications that users recognise and abide by the legal requirements associated with these rights.

- Users may download and print one copy of any publication from the public portal for the purpose of private study or research.

- You may not further distribute the material or use it for any profit-making activity or commercial gain

- You may freely distribute the URL identifying the publication in the public portal

Read more about Creative commons licenses: https://creativecommons.org/licenses/

Take down policy

If you believe that this document breaches copyright please contact us providing details, and we will remove

access to the work immediately and investigate your claim.
} 


\title{
Calculation and manipulation of the chirp rates of high-order harmonics
}

\author{
M. Murakami, ${ }^{1}$ J. Mauritsson, ${ }^{1}$ A. L'Huillier, ${ }^{2}$ K. J. Schafer, ${ }^{1}$ and M. B. Gaarde ${ }^{1}$ \\ ${ }^{1}$ Department of Physics and Astronomy, Louisiana State University, Baton Rouge, Louisiana 70803-4001, USA \\ ${ }^{2}$ Department of Physics, Lund Institute of Technology, P. O. Box 118, S-22100 Lund, Sweden
}

(Received 17 September 2004; published 14 January 2005)

\begin{abstract}
We calculate the linear chirp rates of high-order harmonics in argon, generated by intense, $810 \mathrm{~nm}$ laser pulses, and explore the dependence of the chirp rate on harmonic order, driving laser intensity, and pulse duration. By using a time-frequency representation of the harmonic fields we can identify several different linear chirp contributions to the plateau harmonics. Our results, which are based on numerical integration of the time-dependent Schrödinger equation, are in good agreement with the adiabatic predictions of the strong field approximation for the chirp rates. Extending the theoretical analysis in the recent paper by Mauritsson et al. [Phys. Rev. A 70, 021801(R) (2004)], we also manipulate the chirp rates of the harmonics by adding a chirp to the driving pulse. We show that the chirp rate for harmonic $q$ is given by the sum of the intrinsic chirp rate, which is determined by the new duration and peak intensity of the chirped driving pulse, and $q$ times the external chirp rate.
\end{abstract}

DOI: 10.1103/PhysRevA.71.013410

PACS number(s): 32.80.Rm, 42.65.Ky

\section{INTRODUCTION}

High-order harmonics, which can be generated in the interaction between an intense ultrashort pulse and a gas of atoms [1], represent a unique and versatile source of extreme ultraviolet (xuv) radiation. Applications of the harmonic radiation range from xuv pump-probe spectroscopy $[2,3]$ and interferometry [4] to the generation of attosecond pulses, both in the form of isolated attosecond bursts [5] and in the form of trains of attosecond pulses [6-9]. A characteristic feature of the harmonic pulses is that they are generated with a time-varying phase and therefore exhibit a time-dependent frequency $[10,11]$.

Much effort has been put into the manipulation and characterization of this time-dependent frequency [10-13]. Its manipulation is important for the use of the harmonic radiation as an xuv source since, in addition to its effect on the spectrum of the individual harmonic, the time-dependent frequency and its variation with harmonic order strongly influence the time structure of the attosecond pulse trains generated by superposing a number of harmonics [14]. The characterization of the time-dependent frequency is also of fundamental interest, since the time dependence of the harmonic phase to a large part is intrinsic to the generation process, originating in the electron dynamics of each atom driven by the strong field [15]. The time-dependent frequency therefore yields important information about the harmonic generation process itself. Experimentally, other factors such as ionization-induced blueshifting and phase matching also affect the chirp rates [12], but these can be kept to a minimum by keeping the peak intensity below saturation [13].

The time-dependent frequencies of the harmonics and their variation with harmonic order can be understood in the framework of the semiclassical model of harmonic generation $[16,17]$, in which an electron is released into the continuum via tunnel ionization, accelerated by the laser field, and on returning to the ion core and recombining can transfer its kinetic energy to radiation. In this model, the energy and phase of the emitted light are determined by the electron's kinetic energy and its time of return, respectively. The timedependent phase $\phi_{q}(t)$ is proportional to the laser intensity $I(t), \phi_{q}(t)=-\alpha_{q}^{i} I(t)$, where the phase coefficient $\alpha_{q}^{i}$ is characteristic of the space-time quantum path $i$ the electron has followed [18]. The temporal variation discussed above refers to the slow variation of the pulse envelope. In this adiabatic limit, the time-dependent frequency $\omega_{q}(t)=-d \phi_{q}(t) / d t$ is determined by the cycle to cycle variation of the intensity envelope. The time-dependent frequency is approximately linear close to the peak of the laser pulse, and is often characterized by its linear chirp rate $b_{q}^{d i p}$ :

$$
b_{q}^{d i p} \propto-\alpha_{q}^{i} I_{p e a k} / \tau^{2} .
$$

Here $I_{\text {peak }}$ and $\tau$ are the peak intensity and the full width at half maximum (FWHM) duration, respectively, of the driving pulse. The proportionality constant depends on the pulse shape [19]. In the adiabatic picture, the chirp rate thus scales linearly with the pulse peak intensity, and quadratically with the inverse of the pulse duration. The variation of the chirp rate from harmonic to harmonic originates in the variation of the return time, and thereby $\alpha_{q}^{i}$, with order $[7,8,14]$.

In this paper we theoretically explore the time-frequency (TF) behavior of high-order harmonics, generated in argon by $810 \mathrm{~nm}$ laser pulses with durations ranging from $13 T_{1}$ to $36 T_{1}$, where $T_{1}$ is the laser period. Using a time-frequency analysis, we can resolve more than one linear chirp contribution for the harmonics in the plateau. We calculate the linear chirp rate as a function of the harmonic order and find results that are in good agreement with the predictions based on the strong field approximation (SFA) $[15,18]$ and with recent measurements [13]. In agreement with previous results [20], we find that the most important contributions to the plateau harmonics come from the first (shortest) and the third and longer quantum paths. Finally, as was found experimentally in [13], we show that the harmonic chirp rate $b_{q}$ can 
be manipulated by adding a linear chirp with rate $b_{1}$ to the driving laser pulse according to

$$
b_{q}=q b_{1}+b_{q}^{d i p},
$$

when one takes into account the change in the driving pulse duration and peak intensity caused by its chirping.

The paper is outlined as follows. In Sec. II we discuss our theoretical methods. Sections III and IV present the TF results and the chirp manipulation results, respectively. Section $\mathrm{V}$ summarizes our conclusions.

\section{THEORETICAL METHOD}

We solve the time-dependent Schrödinger equation (TDSE) by numerical integration within the single-activeelectron approximation. We follow exactly the same procedure as outlined in detail in $[17,21,22]$ : the initial state of the atom (the ground state) is found as the solution to the fieldfree time-independent Schrödinger equation, and the timedependent one-electron wave function is then calculated by direct numerical integration of the TDSE in the combined atomic and laser potentials. The atom is described by a pseudopotential, the construction of which is based on Hartree-Slater calculations and is discussed in detail in [23-25]. We use a Gaussian pulse shape with a FWHM duration of $\tau$ and integrate from $t=-2.5 \tau$ to $t=2.5 \tau$, evaluating the time-dependent acceleration form $a(t)$ of the dipole moment in each time step. The dipole spectrum $d(\omega)$ is proportional to the Fourier transform of $a(t)$. We find the time profile $E_{q}(t)$ of harmonic $q$ by multiplying $d(\omega)$ with a window function centered around the $q$ th harmonic and inverse Fourier transforming to the time domain. The results presented below have been obtained with a square window of width $2 \omega_{1}$, where $\omega_{1}$ is the driving frequency, but they do not depend on the shape of the window function.

We are interested in the TF behavior of $E_{q}(t)$ $=\left|E_{q}(t)\right| \exp \left[i \Phi_{q}(t)\right]+$ c.c., where $\Phi_{q}(t)$ is now the full timedependent phase of the $q$ th harmonic electric field. The harmonics in the cutoff region exhibit a simple TF behavior which can be characterized by the linear chirp rate $b_{q}$ of the time-dependent frequency $\omega_{q}(t)=-d \Phi_{q}(t) / d t$. We find the chirp rate by fitting a straight line to $\omega_{q}(t)$ over approximately the FWHM duration of the harmonic pulse (since the harmonic pulses are far from Gaussian in shape, the duration over which the frequency is fitted varies somewhat from harmonic to harmonic). The harmonics in the plateau region have contributions from several quantum paths each with their own TF characteristics. We therefore need to simultaneously represent the temporal and spectral characteristics of these harmonics. We choose the following TF representation (TFR):

$$
S_{q}(t, \omega)=\left|\int d t^{\prime} e^{i \omega t^{\prime}} E_{q}\left(t^{\prime}\right) E_{I R}\left(t^{\prime}-t\right)\right|^{2},
$$

where the probe pulse $E_{I R}\left(t^{\prime}-t\right)$ has a center frequency of $\omega_{1}$ and a duration shorter than that of $E_{q}(t)$. When the delay between the probe and the harmonic pulses is varied, the spectrogram $S_{q}(t, \omega)$ traces how the "instantaneous" spec-

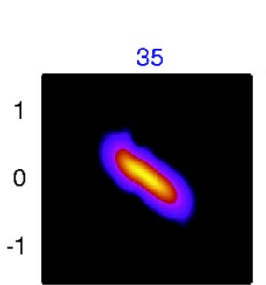

29

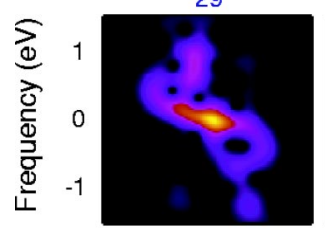

23

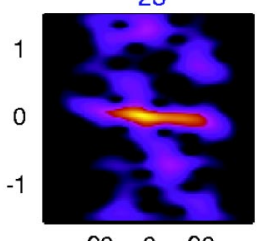

$-20 \quad 0 \quad 20$
Harmonic order

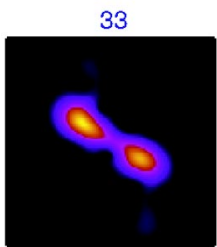

27

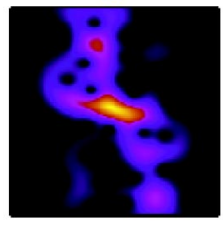

21

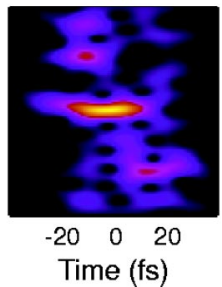

31

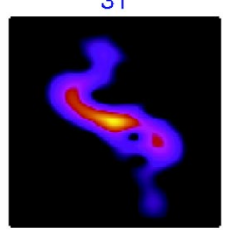

25

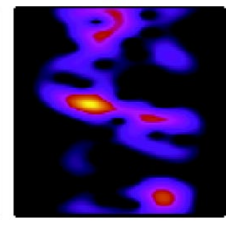

19

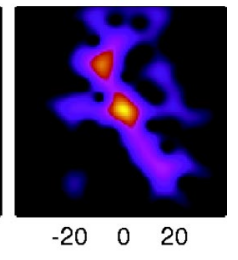

FIG. 1. (Color online) The time-frequency representation of nine consecutive harmonics in argon, driven by a laser pulse with a peak intensity of $2 \times 10^{14} \mathrm{~W} / \mathrm{cm}^{2}$, and a pulse duration of $20 T_{1}$, starting with the harmonic closest to the cutoff energy and moving downward in photon energy. The probe pulse duration used in Eq. (3) is $5 T_{1}$. The TFR is shown in false colors/gray scale. The scale is different from harmonic to harmonic due to the large difference in strength between the plateau and cutoff harmonics.

trum of the harmonic field changes during the pulse. If $E_{q}(t)$ can be characterized by a linear chirp, for instance, then $S_{q}(t, \omega)$ is distributed along a straight line in the $t$ - $\omega$ plane whose slope yields the chirp rate when corrected for the finite duration of the probe pulse [11]. In case $E_{q}(t)$ has multiple spectral contributions with different chirp rates, its TFR will split up into several linear structures, from which we can find the chirp rates one by one. The spectrogram can therefore in many cases resolve the different $\mathrm{TF}$ behaviors due to the different quantum path contributions [19]. We note that our choice of TFR is very close to the experimental approach for the TF characterization of harmonics through the generation of sidebands, discussed in detail in [13].

\section{LINEAR CHIRP RATES OF PLATEAU AND CUTOFF HARMONICS}

The results in this section have been obtained in argon driven by $810 \mathrm{~nm}$ laser pulses with a FWHM duration of $20 T_{1}$ (54 fs) and a peak intensity of $2 \times 10^{14} \mathrm{~W} / \mathrm{cm}^{2}$. The cutoff energy predicted for this system is $36 \omega_{1}(55 \mathrm{eV})$ [26]. To calculate the TFR, we have used a probe pulse with a FWHM duration of $5 T_{1}$.

In Fig. 1, we plot the TFR of nine consecutive harmonics spanning part of the plateau and the cutoff region to make the following observations. The TFR of the harmonics beyond the cutoff is distributed along one direction only, indi- 


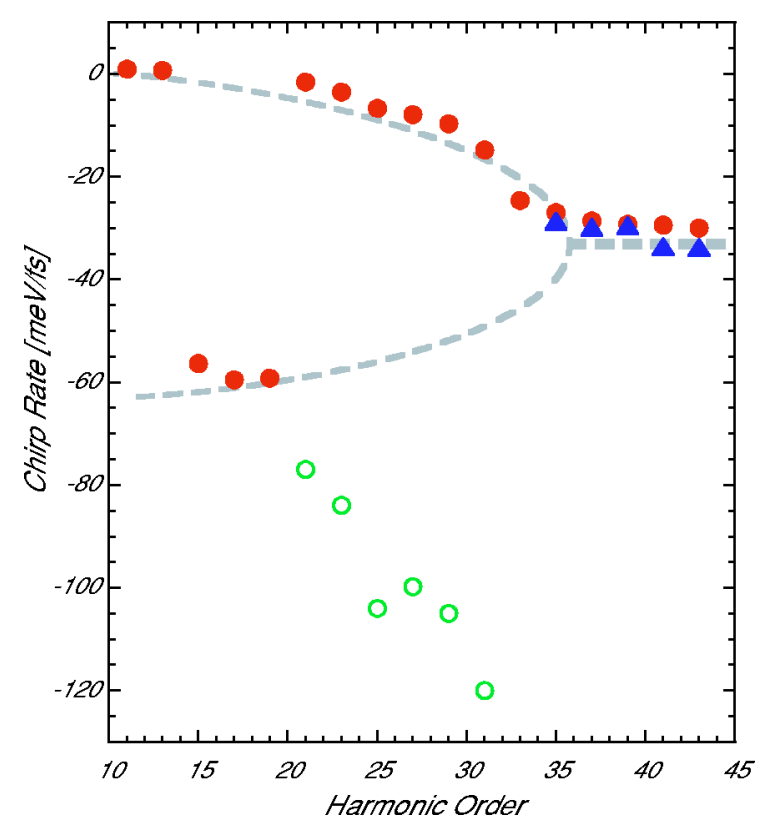

FIG. 2. (Color online) Chirp rates of the high harmonic fields in argon, obtained from the strongest component of TFRs (filled circles), or by direct differentiation (triangles). The SFA predictions for the chirp rates originating in the first two quantum paths are shown with dotted curves. As illustrated in Fig. 1, an additional contribution to the TFR of harmonics is visible in the upper plateau region, from which we have found another set of chirp rates, shown with open circles. These chirp rates originate in the phase behavior of the third quantum path; see [20].

cating that these fields have a unique chirp rate. This chirp rate is independent of order. For the harmonics in the plateau, there are two significant directions for the TFR to spread, one associated with a small negative chirp, and the other with a much larger negative chirp. The slopes of both of these contributions clearly vary from harmonic to harmonic. We have determined these chirp rates separately and plot them in Fig. 2.

The closed circles in Fig. 2 show the chirp rates versus the harmonic energy, found from the primary contributions to the TFR. To calculate them, we find the frequency $\omega$ at which $S_{q}(t, \omega)$ takes its maximum value for each $t$, and then perform a linear fit to this set of points $\omega(t)$. For harmonics beyond the cutoff, we also plot the rates found by direct differentiation of the time-dependent phase of $E_{q}(t)$ (shown with triangles). These are in excellent agreement with the rates extracted from the TFR. The open circles in Fig. 2 have been found from the secondary linear structures in the TFR.

We interpret our results within the framework of the semiclassical model $[14,15,18]$. The TFR resolves the different quantum path contributions to the time-dependent frequency, and gives an intuitive measure of the electron dynamics at the single-atom level. Quantitatively, the chirp rates are in good agreement with predictions of the SFA (dotted curves) for the two shortest quantum paths. In agreement with results of our earlier work, and in contrast to the predictions of the SFA, we also find that (i) the shortest quantum path is dominant for most of the harmonics in argon, and (ii) it is in general the trajectories with return times longer than one ir cycle which yield the next largest contribution [20]. For the harmonics in the lower plateau region, the contributions from the second and the third quantum paths are not distinguishable with our resolution (see also [20]). In an experiment it is possible to spatially separate the contribution from the shortest trajectory from other contributions since its spatial divergence is smaller by approximately an order of magnitude $[8,27]$. In addition, as also seen in Fig. 1, the spectral distribution of the contributions from the longer trajectories is very wide, often making their experimental observation difficult.

\section{MANIPULATION OF THE CHIRP RATES}

Next, we manipulate the harmonic chirps by adding a chirp $b_{1}$ to the driving pulse. In the adiabatic limit, we then expect the harmonics to exhibit a chirp given by the sum of the intrinsic chirp and $q$ times the fundamental chirp. Recent experimental and theoretical results [13] have supported this expectation. In Fig. 3 we detail our theoretical findings in [13]. We concentrate on the harmonics in the cutoff region for which we can directly find the linear chirp rates from the time-dependent phase, as described above.

The chirp is added as it would be in an experiment, by stretching the driving pulse to be positively or negatively chirped, in a way that preserves the pulse energy. This means that the peak intensity of the chirped pulse decreases as

$$
I_{c}=I_{0} \frac{\tau_{0}}{\tau_{c}}
$$

when the pulse is stretched from its original duration $\tau_{0}$ to a duration $\tau_{c}$. We use a duration of the unchirped driving pulse of $\tau_{0}=13 T_{1}$ (35 fs). In Fig. 3(a) the driving pulse has been positively chirped to a FWHM duration of $\tau_{c}=36 T_{1}$. This leads to a linear chirp rate of $b_{1}=+0.50 \mathrm{meV} / \mathrm{fs}$ and a reduced peak intensity of $I_{c}=0.72 \times 10^{14} \mathrm{~W} / \mathrm{cm}^{2}$. The linear chirp rate of the odd harmonics 19 through 27 (the cutoff energy is $19.5 \omega_{1}$ ) found from the time-dependent phase is shown with filled circles. The adiabatic prediction for the chirp rate of the cutoff harmonics as given by Eqs. (2) and (1), using $\tau_{c}$ and $I_{c}$, is shown with open circles. The filled triangles show $q b_{1}$. The harmonics in Fig. 3(b) have been calculated with a negatively chirped pulse with rate $b_{1}=$ $-0.68 \mathrm{meV} / \mathrm{fs}$, a stretched pulse duration of $\tau_{c}=23 T_{1}$, and reduced peak intensity $I_{c}=1.13 \times 10^{14} \mathrm{~W} / \mathrm{cm}^{2}$ (with a cutoff energy of $\left.24.8 \omega_{1}\right)$. In both calculations, the adiabatic prediction is in very good agreement (to within 10\%) with the full calculation. In an experiment, one would also expect the measured chirp rates to be influenced by ionization and phase matching although these can be minimized by the choice of parameters [13].

It is worth noting that when the driving pulse is chirped in this way, which is experimentally the most straightforward approach, the change in the harmonic chirp rate is twofold. In addition to the externally added chirp, the rate of the intrinsic chirp of the cutoff harmonics decreases with the third power of the pulse duration [see Eqs. (1) and (4)]. In addition, the cutoff energy is lowered due to the smaller peak 

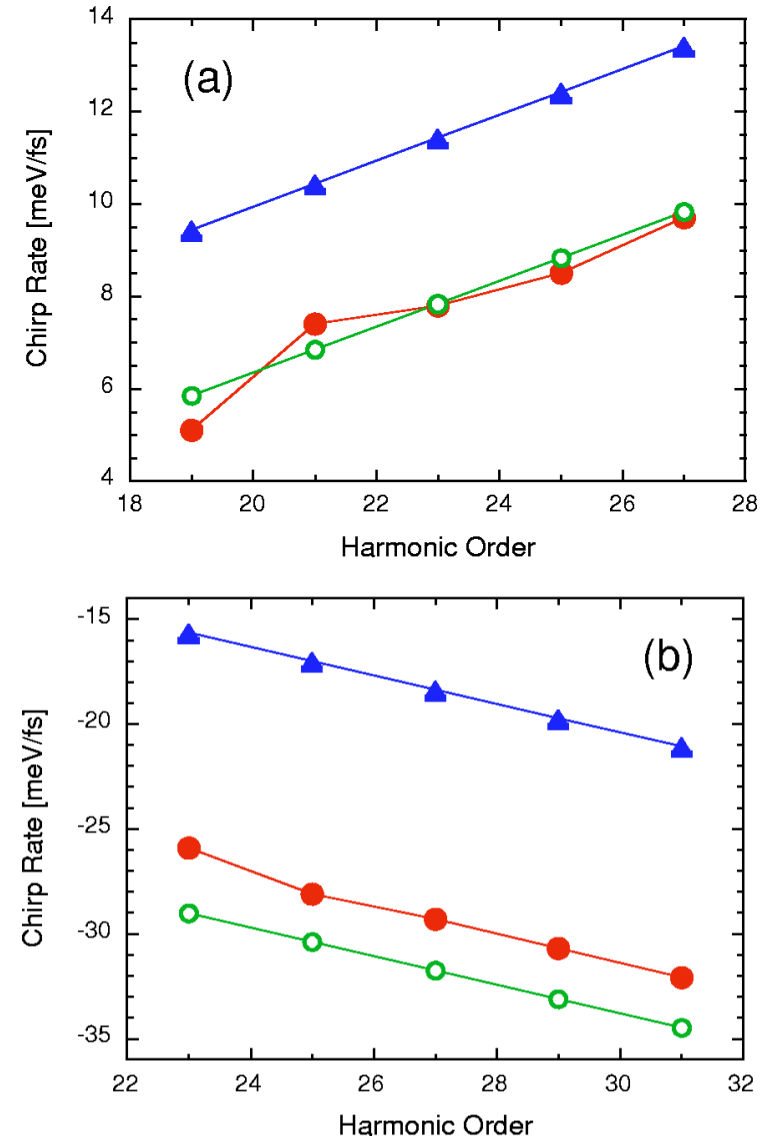

FIG. 3. (Color online) The chirp rates of cutoff harmonics controlled by a driving pulse which has been chirped by stretching from an initial duration of $13 T_{1}$ (see also text). In (a) the driving pulse is positively chirped with a rate of $b_{1}=0.50 \mathrm{meV} / \mathrm{fs}$ to a duration of $36 T_{1}$. This reduces the peak intensity to 0.72 $\times 10^{14} \mathrm{~W} / \mathrm{cm}^{2}$. The driving pulse in (b) has a negative linear chirp rate of $b_{1}=-0.68 \mathrm{meV} / \mathrm{fs}$, a pulse duration of $23 T_{1}$, and a peak intensity of $1.13 \times 10^{14} \mathrm{~W} / \mathrm{cm}^{2}$. Filled circles show the calculated chirp rates; open circles show the adiabatic SFA prediction for the chirp rates. The external chirp $q b_{1}$ is shown with triangles.

intensity. Controlling the chirp of particular harmonics is thus a balance between several factors.

In Fig. 4 we use this manipulation to eliminate the chirp of a cutoff and a plateau harmonic. In (a) we show the TFR of the 35th harmonic calculated with the same parameters as in Fig. 1, a pulse duration of $20 T_{1}$ and a peak intensity of $2 \times 10^{14} \mathrm{~W} / \mathrm{cm}^{2}$. Its linear chirp rate is $-27 \mathrm{meV} / \mathrm{fs}$ (see Fig. 2). To compensate its chirp, we simply add a positive linear chirp with rate $b_{1}=b_{35} / 35=0.77 \mathrm{meV} / \mathrm{fs}$ to the driving pulse, keeping the same intensity and pulse duration. This

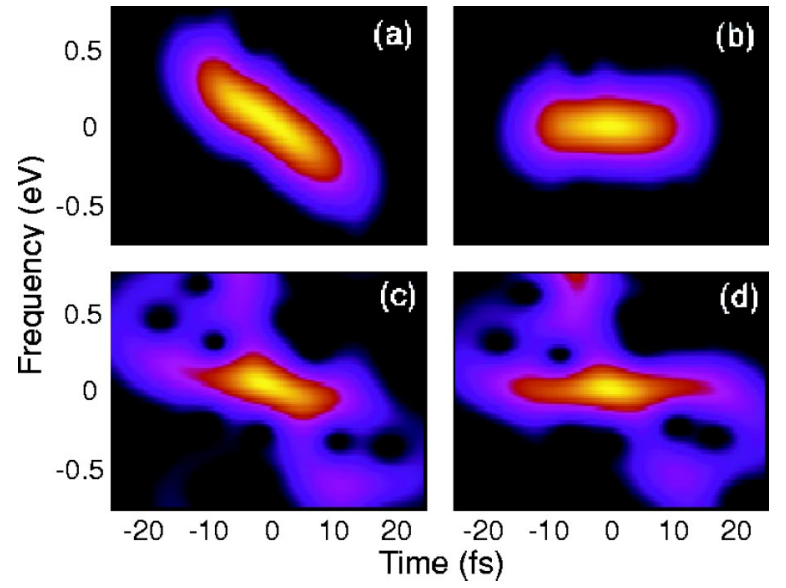

FIG. 4. (Color online) (a) and (c): The TFRs of the 35th and 27th harmonic, respectively, driven by an unchirped pulse as in Fig. 1 (peak intensity $2 \times 10^{14} \mathrm{~W} / \mathrm{cm}^{2}$ and pulse duration $20 T_{1}$ ). In (b) and (d) we show the 35 th and 27 th harmonics, respectively, generated by chirped pulses with the same peak intensity and pulse duration, with positive chirp rates $b_{1}=0.77$ and $b_{1}=0.31 \mathrm{meV} / \mathrm{fs}$.

means that we add bandwidth to the driving pulse. Experimentally, this could be achieved by positive stretching of a shorter pulse $\left(12.6 T_{1}\right)$ to the $20 T_{1}$ duration used here. The resulting TF behavior, which is now flat, is shown in (b). Since the intrinsic chirp of the harmonic has been compensated, the bandwidth of the harmonic is smaller than in (a) even though the bandwidth of the driving pulse is larger [10].

The plateau harmonics have several contributions with different chirps, which means that adding a single chirp to the driving pulse does not compensate the entire TF dependence. Here we manipulate the chirp of the strongest contribution to the plateau harmonics, from the short quantum path. The TFR of the 27th harmonic calculated as in Fig. 4(a) is shown in 4(c). In 4(d) we have added a positive chirp of $b_{1}=0.31 \mathrm{meV} / \mathrm{fs}$ to the driving pulse, again keeping the same pulse duration and peak intensity. This essentially eliminates the chirp of the dominant contribution, but does little (relatively) to the larger chirp.

While eliminating the chirp of the 27th harmonic for part in Fig. 4(d), the manipulation also affects its neighboring harmonics. In Fig. 5 we show the chirp rates of harmonics 23 through 31 generated by the chirped driving pulse of Fig. 4(d). The chirps of all the harmonics have been significantly reduced, except for the 31st harmonic. This can be understood by looking at the order dependence of the chirp rate for these harmonics shown in Fig. 2. The chirp rate due to the first quantum path increases almost linearly in magnitude between harmonics 23 and 29. This is nearly compensated by

31

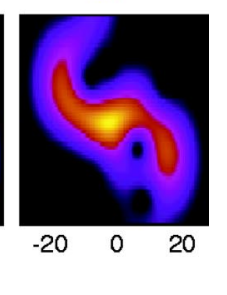

FIG. 5. (Color online) The TFRs of the odd harmonics 23 through 31 , generated by the positively chirped driving pulse in Fig. 4(d). This chirp rate compensates the intrinsic chirp of the 27th harmonic. 
the increase in the external chirp rate with order $\left(q \times b_{1}\right)$. The 31 st harmonic has both a larger intrinsic chirp rate from the first quantum path and a larger contribution from the longer quantum paths (see Fig. 1). The manipulated 31st harmonic shown in Fig. 5 certainly exhibits two different TF behaviors.

\section{SUMMARY}

We have analyzed the TF behavior of many high-order harmonics in argon. Using a TF analysis we could identify several contributions with different linear chirps to the harmonic time profiles. Our results, which are based on numerical integration of the TDSE using a realistic pseudopotential for the one-electron argon atom, are in good agreement with SFA predictions for the harmonic chirp rates. By adding a chirp to the driving pulse we manipulated the harmonic chirp rates and showed that, at least in the adiabatic regime as in this study, the harmonic chirp rates are the sum of their intrinsic and external chirp rates. When the driving pulse is chirped by stretching, the increase in the pulse duration rapidly decreases the intrinsic chirp. Controlling the chirp of any particular harmonic is thus a balance between pulse duration, intensity, and external chirp rate.

\section{ACKNOWLEDGMENTS}

The authors thank P. Johnsson, R. López-Martens, and K. Varjú for stimulating discussions. M.M. and M.G. acknowledge the support of the Louisiana Board of Regents through Grant No. LEQSF(2004-07)-RD-A-09. J.M. and K.S. acknowledge the support of the National Science Foundation through Grant No. PHY-9733890. A.L. acknowledges the support of the Swedish Science Council.
[1] M. Ferray, A. L'Huillier, X. F. Li, L. A. Lompre, G. Mainfray, and C. Manus, J. Phys. B 21, L31 (1988).

[2] J. Larsson, E. Mevel, R. Zerne, A. L'Huillier, C.-G. Wahlström, and S. Svanberg, J. Phys. B 28, L53 (1995).

[3] L. Nugent-Glandorf, M. Scheer, D. A. Samuels, V. Bierbaum, and S. R. Leone, Rev. Sci. Instrum. 4, 1875 (2002).

[4] D. Descamps, C. Lyngå, J. Norin, A. L'Huillier, C.-G. Wahlström, J. F. Hergott, H. Merdji, P. Salières, M. Bellini, and T. W. Hänsch, Opt. Lett. 25, 135 (2000).

[5] M. Hentschel, R. Kienberger, Ch. Spielmann, G. A. Reider, N. Milosevic, T. Brabec, P. Corkum, U. Heinzmann, M. Drescher, and F. Krausz, Nature (London) 414, 509 (2001).

[6] P. M. Paul, E. S. Toma, P. Breger, G. Mullot, F. Augé, Ph. Balcou, H. G. Muller, and P. Agostini, Science 292, 1689 (2001).

[7] Y. Mairesse, A. de Bohan, L. J. Frasinski, H. Merdji, L. C. Dinu, P. Monchicourt, P. Breger, M. Kovačev, R. Taïeb, B. Carré, 1, H. G. Muller, P. Agostini, and P. Salières, Science 302, 1540 (2003).

[8] R. López-Martens, K. Varjú, P. Johnsson, J. Mauritsson, Y. Mairesse, P. Salières, M. B. Gaarde, K. J. Schafer, A. Persson, S. Svanberg, C.-G. Wahlström, and A. L'Huillier, Phys. Rev. Lett. (to be published).

[9] Ph. Antoine, A. L'Huillier, and M. Lewenstein, Phys. Rev. Lett. 77, 1234 (1996).

[10] Z. Chang, A. Rundquist, H. Wang, I. Christov, H. C. Kapteyn, and M. M. Murnane, Phys. Rev. A 58, R30 (1998).

[11] J. Norin, J. Mauritsson, A. Johansson, M. K. Raarup, S. Buil, A. Persson, O. Duhr, M. B. Gaarde, K. J. Schafer, U. Keller, C.-G. Wahlström, and A. L'Huillier, Phys. Rev. Lett. 88, 193901 (2002).

[12] T. Sekikawa, T. Kanai, and S. Watanabe, Phys. Rev. Lett. 91,
103902 (2003).

[13] J. Mauritsson, P. Johnsson, R. López-Martens, K. Varjú, W. Kornelis, J. Biegert, U. Keller, M. B. Gaarde, K. J. Schafer, and A. L'Huillier, Phys. Rev. A 70, 021801(R) (2004).

[14] K. Varjú, Y. Mairesse, M. B. Gaarde, P. Johnsson, S. Kazamias, J. Mauritsson, K. J. Schafer, P. Balcou, A. L'Huillier, and P. Salières, J. Mod. Opt. (to be published).

[15] M. Lewenstein, P. Salières, and A. L'Huillier, Phys. Rev. A 52, 4747 (1995).

[16] P. B. Corkum, Phys. Rev. Lett. 71, 1994 (1993).

[17] K. C. Kulander, K. J. Schafer, and J. L. Krause, in Atoms in Intense Laser Fields, edited by M. Gavrila (Academic Press, San Diego, 1992).

[18] M. Lewenstein, Ph. Balcou, M. Yu. Ivanov, A. L'Huillier, and P. B. Corkum, Phys. Rev. A 49, 2117 (1994).

[19] M. B. Gaarde, Opt. Express 8, 529 (2001).

[20] M. B. Gaarde and K. J. Schafer, Phys. Rev. A 65, 031406R (2002).

[21] K. J. Schafer and K. C. Kulander, Phys. Rev. Lett. 78, 638 (1997).

[22] J. L. Krause, K. J. Schafer, and K. C. Kulander, Phys. Rev. A 45, 4998 (1992).

[23] K. C. Kulander and T. N. Rescigno, Comput. Phys. Commun. 63, 523 (1991).

[24] K. C. Kulander, Phys. Rev. A 38, 778 (1988).

[25] K. C. Kulander, K. J. Schafer, and J. L. Krause, Laser Phys. 3, 359 (1993).

[26] J. L. Krause, K. J. Schafer, and K. C. Kulander, Phys. Rev. Lett. 68, 3535 (1992).

[27] M. Bellini, M. B. Gaarde, T. W. Hänsch, A. L'Huillier, C. Lyngå, A. Tozzi, and C.-G. Wahlström, Phys. Rev. Lett. 81, 297 (1998). 